\title{
Relationship between epigenetic changes in Wnt antagonists and acute leukemia
}

\author{
HUA-RONG ZHOU, HAI-YING FU, DAN-SEN WU, YUAN-YUAN ZHANG, SI-HAN HUANG, \\ CONG-JIE CHEN, JIAN-GUO YAN, JIN-LONG HUANG and JIAN-ZHEN SHEN

\begin{abstract}
Fujian Institute of Hematology, Fujian Provincial Key Laboratory of Hematology, Fujian Medical University Union Hospital, Fuzhou, Fujian 350001, P.R. China
\end{abstract}

Received November 8, 2016; Accepted December 16, 2016

DOI: $10.3892 /$ or.2017.5509

\begin{abstract}
The present study was designed to investigate the relationship among epigenetic changes in Wnt antagonists, histone H4K20mel and the expression of tumor-suppressor genes in acute leukemia (AL) to better understand the pathogenesis of leukemia. Quantitative reverse transcription polymerase chain reaction (qRT-PCR) was performed to detect messenger RNA (mRNA) expression levels of Wnt antagonists (Wnt5a, HDPR1, DKK1 and DKK3) in patients with AL and in normal controls; pyrophosphate sequencing was performed to detect the methylation status of the Wnt5a promoter; and western blotting was performed to detect the overall expression levels of Wnt5a protein and histone H4K20me1 in patients with acute myeloid leukemia (AML) and in normal controls. The relationship between Wnt5a protein expression and histone H4K20me1 was analyzed. Chromatin immunoprecipitation-qPCR (ChIP-qPCR) was performed to investigate the recruitment of H4K20mel and SET8 to the Wnt5a promoter and coding regions. Our results demonstrated that the expression levels of Wnt antagonists were generally low in AML, but showed differential expression
\end{abstract}

Correspondence to: Dr Jian-Zhen Shen, Fujian Institute of Hematology, Fujian Provincial Key Laboratory of Hematology, Fujian Medical University Union Hospital, 29 Xin-Quan Road, Fuzhou, Fujian 350001, P.R. China

E-mail: doctorsjz@163.com

Abbreviations: AML, acute myeloid leukemia; ALL, acute lymphocytic leukemia; AL, acute leukemia; M0, acute myeloid leukemia with minimal differentiation; M1, AML with partial differentiation; M2, AML with maturation; M3, acute promyelocytic leukemia; M4, acute myelomonocytic leukemia with granules; M5, acute monocytic leukemia; M6, acute erythroleukemia; NC normal control; qRT-PCR, quantitative reverse transcription-polymerase chain reaction; ChIP-qPCR, chromatin immunoprecipitation-qPCR; SET, KMT5A/SETD8/Pr-Set7; PBS, phosphate-buffered saline; BCA, bicinchoninic acid; SDS-PAGE, sodium dodecyl sulphate-polyacrylamide gel electrophoresis

Key words: Wnt antagonists, epigenetics, histone modification, H4K20me1, acute leukemia in acute lymphocytic leukemia (ALL). In most cases of AML, methylation of the Wnt5a promoter was observed and Wnt5a protein expression was low. In some cases of AML, the overall level of H4K20me1 protein was higher than that in normal controls. In addition, Wnt5a expression was positively correlated with $\mathrm{H} 4 \mathrm{~K} 20 \mathrm{me} 1$ expression and was unrelated to the methylation status of its promoter. Moreover, H4K20me1 and SET8 were enriched in the Wnt5a promoter region and coding region. By contrast, Wnt5a expression was unrelated to H4K20me1 expression in normal controls. Moreover, we observed that the methylation of Wnt antagonists was often found in patients with AL, particularly those with AML, whereas the extent of methylation was variable in ALL patients. Wnt5a expression was positively correlated with the enrichment of H4K20me1 and SET8 at the Wnt5a promoter and coding regions. H4K20me1 increased Wnt5a expression by promoting transcription initiation and elongation.

\section{Introduction}

First proposed by C.H. Waddington in 1957, epigenetics is a branch of genetics that involves the aberrant methylation of tumor-suppressor genes, abnormal histone post-translational modification, non-coding RNA interference and chromatin remodeling. Acute leukemia (AL) often exhibits aberrant epigenetic modifications, such as inactivation of the methylation of Wnt antagonists and excessive activation of histone deacetylase. Therefore, investigating the epigenetic changes in $\mathrm{AL}$ is essential for identifying potential key therapeutic targets.

H4K20 was discovered in 1969 (1), but it was not identified as a major site of methylation until 2001 (2). In multicellular organisms, H4K20 may present 3 different levels of modification: monomethylation, bimethylation and trimethylation. The methylation status is regulated by specific methyltransferases; KMT5A/SETD8/Pr-Set7 (SET8) is the only methyltransferase that catalyzes H4K20 monomethylation (3). H4K20me1 is the precursor of $\mathrm{H} 4 \mathrm{~K} 20 \mathrm{me} 2 / 3$ and is involved in various processes such as DNA damage response, mitosis, chromatin condensation, DNA replication and gene regulation (4-6). Previously, researchers believed that H4K20me1 was a transcription-inhibiting histone (7); however, in 2014, studies revealed that $\mathrm{H} 4 \mathrm{~K} 20$ mel plays an important role in enhancing the transcription and elongation of RNA polymerase II (8). 
To date, no studies have been conducted to investigate the relationship between H4K20me1 and SET8, the expression of tumor suppressor genes, and DNA methylation in leukemia. Our previous study showed that hypermethylation of the promoter region of Wnt5a (a Wnt pathway tumor suppressor gene) was detected in some clinical acute myeloid leukemia (AML) specimens. However, there was no decrease in Wnt5a expression in these specimens, suggesting that hypermethylation had no effect on the expression of suppressor genes. Moreover, the expression of histone H4K20me1 was higher in these specimens than in those with low Wnt5a expression. Therefore, we hypothesized that the expression of H4K20mel and Wnt5a may be related and that H4K20me1 may play a larger role in gene transcription than methylation status. This hypothesis may help explain the discordant hypermethylation and inhibition of gene expression observed in clinical specimens, thus providing additional information to the current theory of methylation-induced gene inactivation.

\section{Materials and methods}

Clinical specimens. A total of 95 cases of treatment-naive AL diagnosed and treated at the Affiliated Union Hospital of Fujian Medical University between November 2011 and December 2013 were included in the present study. Among them, 53 patients were male and 42 were female, with a median age of 37 (7-82) years. Moreover, 62 patients had AML and 33 had acute lymphocytic leukemia (ALL) [T cell ALL (ALL-T), $n=11$; B-cell ALL (ALL-B), n=22]. All cases met the diagnostic criteria of the French-American-British (FAB) classification system concerning morphology, immunology, cytogenetics and molecular biology (MICM). A total of 58 bone marrow transplant donors were included in the normal control group (Table I). All patients signed an informed consent form indicating that they agreed to the use of their specimens for research. All specimens were collected from bone marrow.

Collection of bone marrow mononuclear cells. A bone marrow specimen ( $5 \mathrm{ml}$, heparin-treated) was collected, slowly added (with no mixing) to the top of an equal volume of lymphocyte separation medium in a centrifuge tube and centrifuged at room temperature at 2,500 $\mathrm{rpm}$ for $20 \mathrm{~min}$. After centrifugation, the specimen was separated into 4 layers (top to bottom): plasma, mononuclear cells, separation medium, red blood cells and platelets. The mononuclear cell layer was carefully collected, subsequently washed with phosphate-buffered saline (PBS) and centrifuged at 2,000 rpm for $5 \mathrm{~min}$ twice. The supernatant was discarded and the cell pellet was used to extract RNA, proteins or DNA, or stored at $-80^{\circ} \mathrm{C}$ for later use.

RNA extraction and fluorescence qPCR. qRT-PCR was performed to detect Wnt5a, HDPR1, DKK1 and DKK3 expression. TRIzol reagent (Invitrogen Corp., Carlsbad, CA, USA) was used for one-step total RNA extraction from bone marrow specimens according to the manufacturer's instructions. First-strand cDNA was synthesized using an RNA reverse transcription kit (Fermentas Inc., Glen Burnie, MD, USA) according to the manufacturer's instructions. An ABI 7500 fluorescence qPCR instrument and FastStart Universal SYBR-Green Master (ROX) kit were used for
Table I. Age and gender composition of acute leukemia patients and normal controls.

\begin{tabular}{llcc}
\hline \multirow{2}{*}{ Group } & Age (years) & Male (n) & Female (n) \\
\cline { 3 - 4 } & $32.12 \pm 16.21$ & 31 & 31 \\
AML & $42.44 \pm 16.64$ & 22 & 11 \\
ALL & $33.65 \pm 7.37$ & 24 & 34 \\
Normal control & & & Gender \\
\hline
\end{tabular}

AML, acute myeloid leukemia; ALL, acute lymphocytic leukemia.

real-time fluorescence qPCR, using GAPDH as an internal reference; $\mathrm{Ct}$ values were compared to analyze differences in the mRNA expression levels of Wnt5a, and related genes in bone marrow cells between ALL, AML and the normal control group: $\Delta \mathrm{Ct}=\mathrm{Ct}$ value (target primer) $-\mathrm{Ct}$ value (GAPDH), and then the $2^{-\Delta \mathrm{Ct}}$ values were compared. The following specific mRNA primers were used: Wnt5a, 5'-GACCACATGCAGTA CATCGGAGAAG-3' (forward) and 5'-TCCACCTTCGATGT CGGAATTG-3' (reverse); HDPR1, 5'-AAGAGCACCTGGA GACAGACAG-3' (forward) and 5'-GCTGGAATGACAACT GGATAAAC-3' (reverse); DKK1, 5'-CTGCAAAAATGGAA TATGTGT-3' (forward) and 5'-CTTCTTGTCCTTTGGTG TGA-3' (reverse); DKK3, 5'-GATGCCCTTGTGCCAGT-3' (forward) and 5'-TGCCAACTTCATACTCATCGG-3' (reverse); and GAPDH, 5'-GGATGCAGGGATGATGTTCT-3' (forward) and 5'-TGCCACTCAGAAGACTGTGG-3' (reverse).

Western blotting. Cell lysis buffer and a protease inhibitor cocktail were added to the cell pellet, and a bicinchoninic acid (BCA) protein assay kit (Pierce, Rockford, IL USA) was used to determine protein concentration.

For SDS-polyacrylamide gel electrophoresis (SDS-PAGE), the sample volume to be loaded was calculated based on the protein concentration (30 $\mu \mathrm{g}$ protein). Proteins were separated via SDS-PAGE, transferred to a polyvinylidene fluoride membrane, blocked in skim milk at room temperature for $1 \mathrm{~h}$, and incubated with diluted mouse anti-human Wnt5a monoclonal antibody (1:5,000), rabbit anti-human H4K20me1 monoclonal antibody (1:10,000) (both from Millipore, Billerica, MA, USA), or rabbit anti-human GAPDH monoclonal antibody (1:5,000; Abcam, Cambridge, MA, USA) overnight at $4^{\circ} \mathrm{C}$. Next, the membrane was washed with Tris-buffered saline with Tween-20 (TBST) (10 min, x3) and incubated with horseradish peroxidase-labeled goat anti-mouse antibody or goat anti-rabbit secondary antibody for $1 \mathrm{~h}$. An equal volume of reagent $\mathrm{A}$ and $\mathrm{B}$ of an ECL luminescent kit was mixed and evenly spread on the membrane. Subsequently, the membrane was exposed, developed and fixed to Kodak film in the darkroom; Quantity One software (Bio-Rad Laboratories, Hercules, CA USA) was used for the quantitative analysis, using GAPDH as an internal reference. The experiment was repeated 3 times.

Pyrophosphate sequencing. Pyrophosphate sequencing was performed to determine the methylation rate of the $\mathrm{CpG}$ island 
of the Wnt5a promoter in bone marrow cells. PyroMark Assay Design 2.0 was used to design pyrophosphate sequencing primers, which were synthesized by BGI (Shenzhen, China). The following primer sequences were used: Wnt5a, 5'-ATATTTGGGGTTG GAAAGTTTTAA-3' (forward) and 5'-ACCCACAACAAAA ACAAAACCTAATC-3' (reverse); Wnt5a-sequence, GGGTT GGAAAGTTTTAATTAT.

Bone marrow mononuclear cells were collected, and genomic DNA was extracted using phenol-chloroform. An EpiTect Bisulfite kit (Qiagen, Valencia, CA, USA) was used for bisulfite modification and purification of genomic DNA in strict accordance with the manufacturer's instructions. For amplification of sulfated genomic DNA, the total reaction volume was $50 \mu \mathrm{l}$, including $0.2 \mu \mathrm{l}$ of $\operatorname{Taq}(5 \mathrm{U} / \mu \mathrm{l}), 2 \mu \mathrm{l}$ of template, $1 \mu \mathrm{l}$ of dNTPs (10 mM each), $1 \mu \mathrm{l}$ of forward and reverse primer (50 pmol $/ \mu \mathrm{l}), 10 \mu \mathrm{l}$ of $5 \mathrm{X}$ buffer GC (KAPA), and $34.8 \mu \mathrm{l}$ of nuclease-free water. The reaction conditions were as follows: $95^{\circ} \mathrm{C}$ pre-denaturation for $3 \mathrm{~min} ; 94^{\circ} \mathrm{C}$ denaturation for $30 \mathrm{sec}, 50^{\circ} \mathrm{C}$ annealing for $30 \mathrm{sec}$, and $72^{\circ} \mathrm{C}$ extension for $60 \mathrm{sec}$ for 40 cycles; and $72^{\circ} \mathrm{C}$ extension for $7 \mathrm{~min}$. Then, $2 \mu \mathrm{l}$ of reactant haptoglobin, $38 \mu \mathrm{l}$ of binding buffer and $40 \mu \mathrm{l}$ of PCR product were added into a 96-well $\mathrm{PCR}$ reaction plate and mixed at room temperature for $10 \mathrm{~min}$. A vacuum pump was used to draw the haptoglobin and PCR product suspension, which was then successively immersed in $70 \%$ ethanol, $0.2 \mathrm{M} \mathrm{NaOH}$, and washing buffer for $5 \mathrm{sec}$ each. Next, the vacuum pump was turned off and haptoglobin and the PCR product on the probe were placed into $40 \mu \mathrm{l}$ of annealing buffer (containing $1.5 \mu \mathrm{l}$ of sequencing primers), denatured at $85^{\circ} \mathrm{C}$ for $2 \mathrm{~min}$, and cooled to room temperature, allowing annealing and hybridization between the primers and the template. Pyrosequencing design software was used to calculate the dose, and the substrate mixture, enzyme mixture and 4 types of dNTPs were successively added into the reagent compartment, which, along with the 96-well reaction plate, was placed into a pyrosequencing detector (PyroMark Q96 ID) (both from Qiagen) for reaction; Pyro Q-CpG software was used for automatic analysis of the methylation status of each site.

Chromatin immunoprecipitation (ChIP). Three donor bone marrow specimens were collected. Moreover, 3 AML specimens with methylation of the Wnt5a promoter, and 3 AML specimens each with high or low Wnt5a expression were selected. The EZ-Magna ChIP ${ }^{\text {тм }}$ A/G One-Day Chromatin Immunoprecipitation kit (cat no. 17-10086; Millipore) was used for ChIP of bone marrow cells. Cell isolation, sonication, ChIP, eluting and de-crosslinking of protein complexes by immunoprecipitation were performed according to the manufacturer's instructions. Immunoprecipitated DNA was detected with real-time PCR; primers were designed for different regions of the Wnt5a gene, using GAPDH as an internal reference. The following primer sequences were used: Wnt5a promoter region, 5'-GGTCTTTTGCACAATCACGCC-3' (forward) and 5'-TTTCCAACGTCCATCAGCGAC-3' (reverse); Wnt5a coding region, 5'-GATGGCTGGAAGTGCA ATGTCT-3' (forward) and 5'-ACCTGGGCGAAGGAGAAA AA-3' (reverse); and GAPDH, 5'-TACTAGCGGTTTTACGG GCG-3' (forward) and 5'-TCGAACAGGAGGAGCAGAGAG CGA-3' (reverse). The following antibodies were used:
CHIPAb + monomethyl-histone H4 (Lys20) (cat no. 17-651; Millipore), and anti-KMT5A/SETD8/Pr-SET7 antibody, (ab3798; Abcam).

Fluorescence qPCR was performed using DNA fragments from each immunoprecipitation and from each input sample, and the data were analyzed using $\%$ of input and fold-change. Standardized amount of DNA: $\Delta \mathrm{Ct}_{\text {normalized ChIP }}=\mathrm{Ct}_{\mathrm{ChIP}_{\mathrm{P}}}-\left[\mathrm{Ct}_{\text {Input }}\right.$ - $\log _{2(\text { Input Dilution Factor) }}$; Input Dilution Factor $=$ (fraction of the input chromatin $)^{-1}=(1 \%)^{-1}=100 ; \%$ of Input $=2^{-\Delta \mathrm{Ct} \text { normal- }}$ ized ChIP $x 100 \%$; Fold-Change $=2^{-\Delta \Delta \mathrm{Ct}}$, where $\Delta \Delta \mathrm{Ct}=\Delta \mathrm{Ct}$ normalized ChIPtarget group - $\Delta \mathrm{Ct}$ normalized ChIPcontrol group.

Statistical analysis. The data are expressed as the mean \pm SD and were analyzed using SPSS 19.0 software. The data were non-normally distributed. A Mann-Whitney U test was performed for between-group comparisons, and a Chi-square test was performed to compare percentages between 2 groups. $\mathrm{P}<0.05$ was considered to indicate a statistically significant result.

\section{Results}

Wnt5a, HDPR1, DKK1 and DKK3 expression is lower in AML patients than in normal controls. qRT-PCR showed that the relative expression of Wnt5a was lower in bone marrow cells from the AML and ALL ( $\mathrm{T}$ cell) patients than that from the normal controls [AML patients vs. normal controls, $\mathrm{P}<0.0001$; ALL ( $\mathrm{T}$ cell) patients vs. normal controls, $\mathrm{P}=0.0162]$. Furthermore, the relative expression of HDPR1 was lower in bone marrow cells from the AML patients than that from the normal controls (AML patients vs. normal controls, $\mathrm{P}<0.0001$ ) and was higher in the ALL patients than that in the normal controls $(\mathrm{P}=0.00362)$. The relative expression of $\mathrm{DKK} 1$ was lower in bone marrow cells from the AML patients than that from the normal controls $(\mathrm{P}=0.0430)$ and was higher in the ALL patients than that in the normal controls $(\mathrm{P}=0.0016)$. The relative expression of DKK3 was significantly lower in bone marrow cells from the AML and ALL patients than that from the normal controls (AML patients vs. normal controls, $\mathrm{P}<0.0001$; ALL patients vs. normal controls, $\mathrm{P}<0.0001$ ) (Fig. 1).

Western blotting was performed to detect the expression level of the Wnt5a protein. Of the 62 AML cases, Wnt5a expression was detected in 36 cases, and thus, the positive expression rate was $58.06 \%$. Of the 58 normal control samples, Wnt5a expression was detected in 47 cases, and thus, the positive expression rate was $81.03 \%$ (Fig. 2). Consequently, Wnt5a protein expression was lower in bone marrow cells from the AML patients than that from the normal controls (Pearson Chi-square value=7.414; $\mathrm{P}=0.006$ ) (Fig. 3).

DNA methylation rate of the Wnt5a promoter is higher in AML patients than in normal controls. The mean methylation rate of $6 \mathrm{CpG}$ islands in the Wnt5a promoter was determined using $10 \%$ as the cut-off value; that is, methylation was negative when the mean methylation rate was $0-10 \%$ and positive when the mean methylation rate was $\geq 10 \%$. The results revealed that among the 62 AML cases, 33 were positive and 29 were negative for methylation of the Wnt5a promoter (53.23\% positive rate). Among the 58 normal controls, 8 were 
A

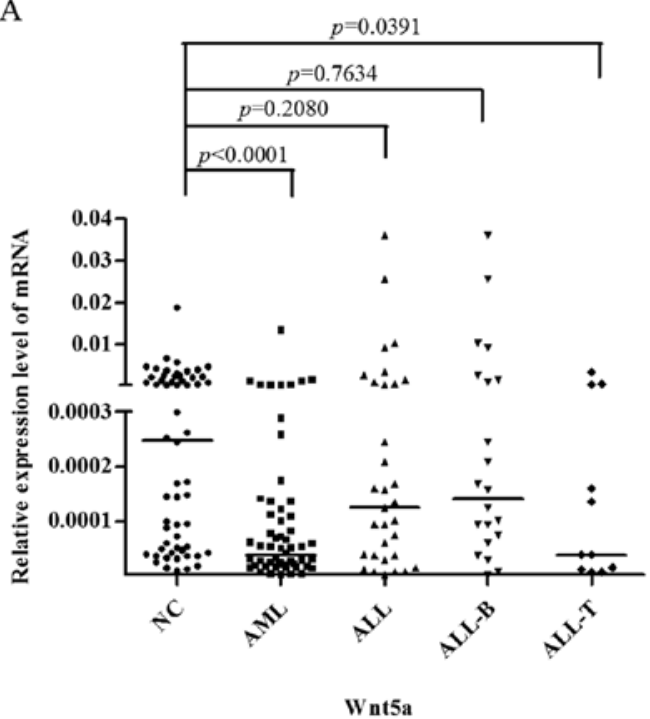

$\mathrm{C}$
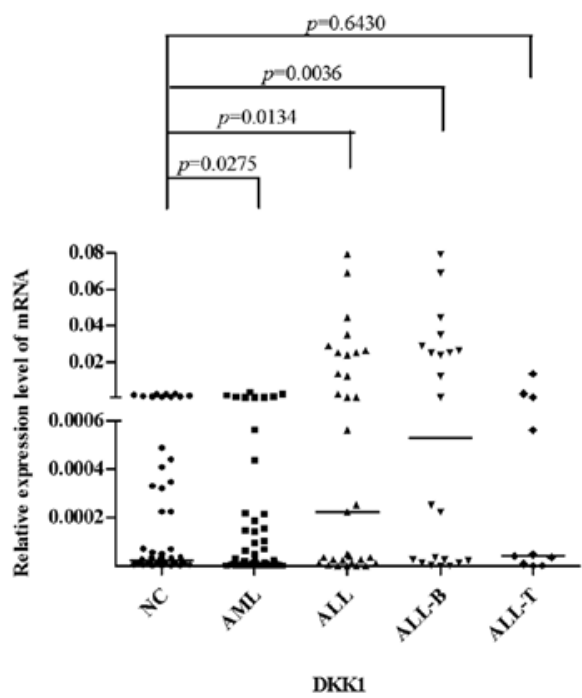

B
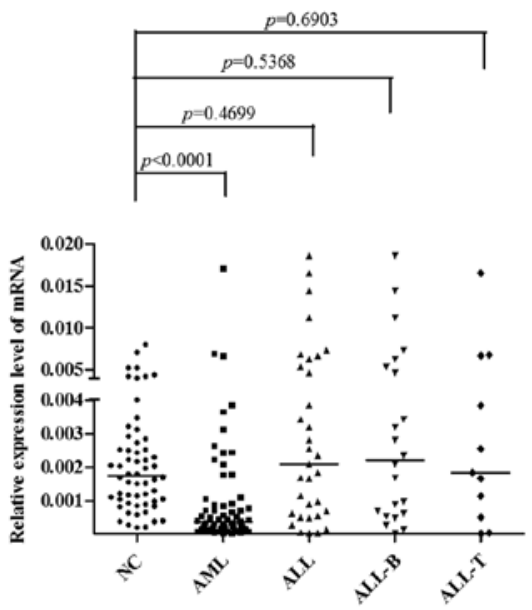

HDPR1

$\mathrm{D}$
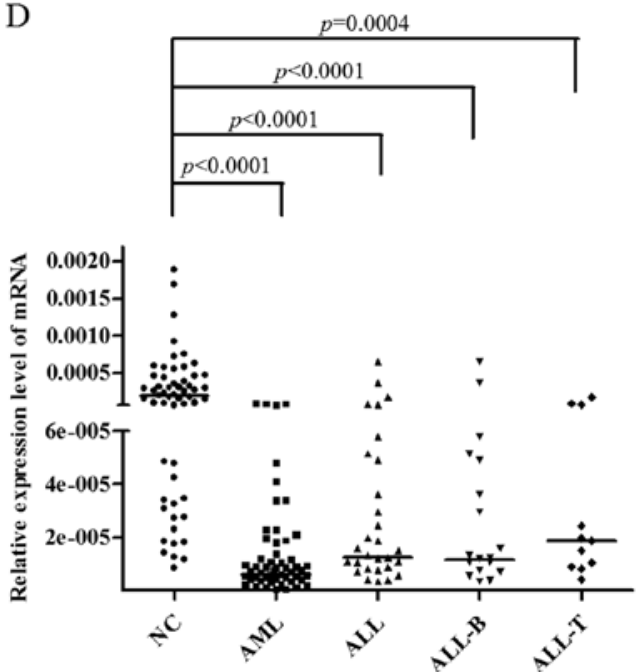

DKK3

Figure 1. Comparison of the mRNA expression of Wnt antagonists (Wnt5a, HDPR1, DKK1 and DKK3) in AML and ALL patients vs. normal controls. (A) The relative expression of Wnt5a was lower in bone marrow cells from the AML and ALL (T cell) patients than that from the normal controls [AML patients vs. normal controls, $\mathrm{P}<0.0001$; ALL (T cell) patients vs. normal controls, $\mathrm{P}=0.0162]$. (B) The relative expression of HDPR1 was lower in bone marrow cells from the AML patients than that from the normal controls (AML patients vs. normal controls, $\mathrm{P}<0.0001$ ) and was higher in the ALL patients than that in the normal controls $(\mathrm{P}=0.00362)$. (C) The relative expression of $\mathrm{DKK} 1$ was lower in bone marrow cells from the AML patients than that from the normal controls $(\mathrm{P}=0.0430)$ and was higher in the ALL patients than that in the normal controls $(\mathrm{P}=0.0016)$. (D) The relative expression of DKK3 was significantly lower in bone marrow cells from AML and ALL patients than from normal controls (AML patients vs. normal controls, $\mathrm{P}<0.0001$; ALL patients vs. normal controls, $\mathrm{P}<0.0001)$. AML, acute myeloid leukemia; ALL, acute lymphocytic leukemia.

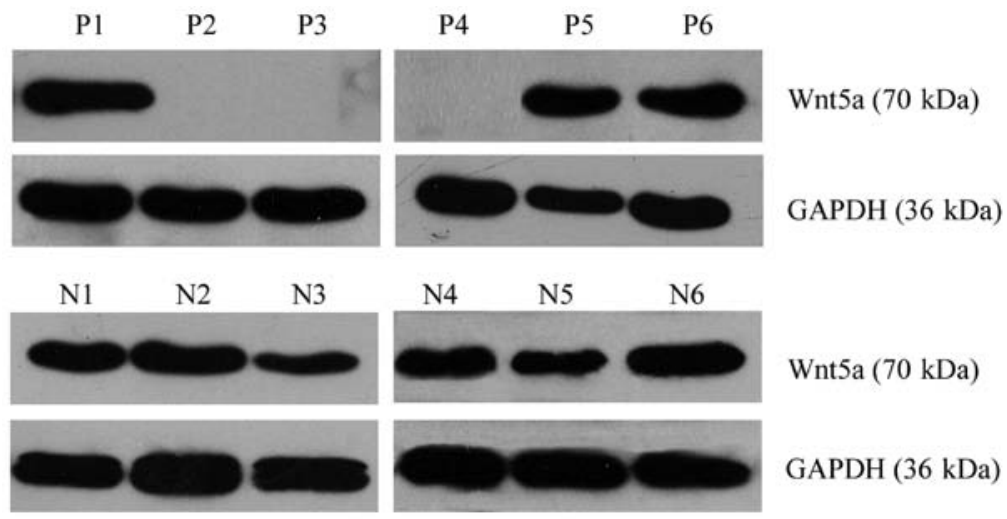

Figure 2. Western blot analysis of Wnt5a expression in bone marrow mononuclear cells from AML patients and normal controls. Wnt5a protein expression was lower in bone marrow cells from the AML patients than that from the normal controls. P1-P6, AML; N1-N6, normal control. AML, acute myeloid leukemia. 


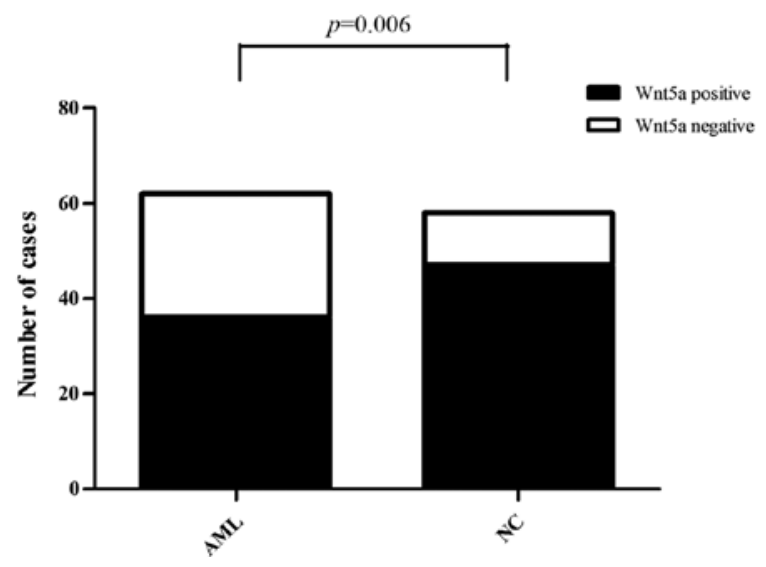

Figure 3. Comparison of Wnt5a protein expression in bone marrow mononuclear cells from AML patients vs. normal controls. The protein expression of Wnt5a was lower in bone marrow cells from the AML patients than from the normal controls (Pearson Chi-square value $=7.414, \mathrm{P}=0.006$ ). AML, acute myeloid leukemia.

positive and 50 were negative for methylation of the Wnt5a promoter (13.79\% positive rate). Statistical analysis revealed that the positive methylation rate of the Wnt5a promoter was significantly higher in the AML patients than that in the normal controls (Fig. 4A and B) (Pearson Chi-square value=20.716, $\mathrm{P}<0.01$; Fig. 5, Table II).

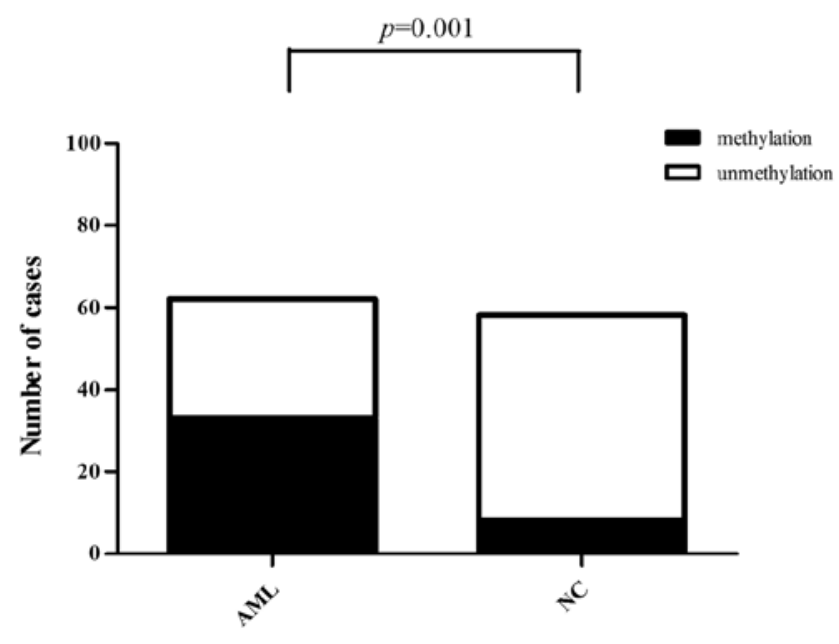

Figure 5. Methylation rate of the Wnt5a promoter in AML patients and normal controls (pyrophosphate sequencing). AML, acute myeloid leukemia.

Overall H4K20mel expression is higher in AML patients than in normal controls. Of the 62 AML cases, H4K20me1 expression was positive in 43 cases and negative in 19 cases (69.35\% positive expression rate). Of the 58 normal controls, H4K20me1 expression was positive in 29 cases and negative in 29 cases $(50.00 \%$ positive expression rate). Consequently,
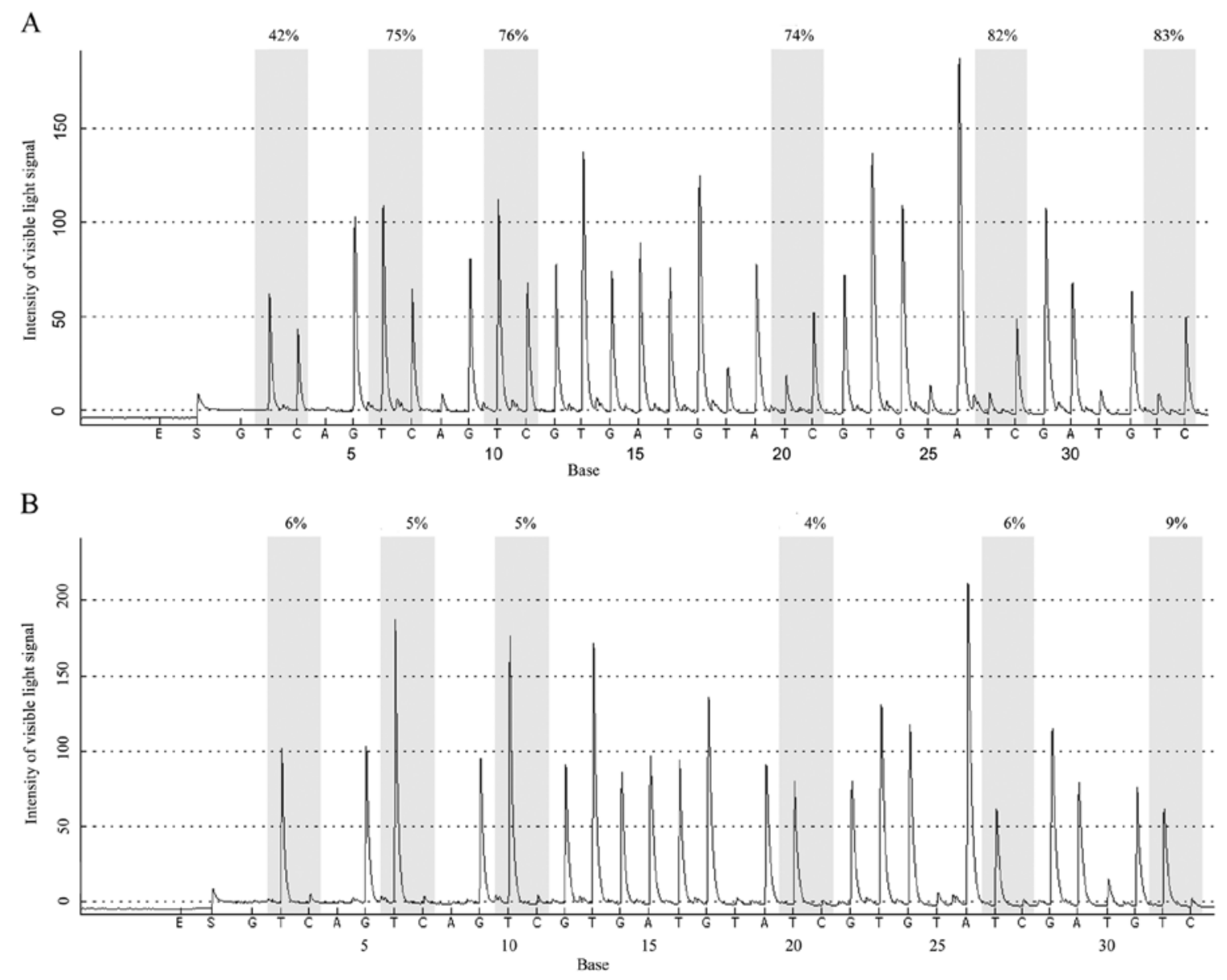

Figure 4. Pyrophosphate sequencing pattern of the Wnt5a promoter in AML patients and normal controls. (A) The CpG islands of the Wnt5a promoter in the AML patients are highly methylated. (B) The CpG islands of the Wnt5a promoter in the AML patients are lowly methylated. AML, acute myeloid leukemia. 
Table II. The methylation rate of CpG islands in the Wnt5a promoter in AML patients (A-C) and normal controls (D-F).

\begin{tabular}{lrrrrrrrr}
\hline & \multicolumn{7}{c}{ Methylation (\%) } \\
\cline { 2 - 6 } Sample ID & Pos. 1 & Pos. 2 & Pos. 3 & Pos. 4 & Pos. 5 & Pos. 6 & No. of included CpGs & Mean (\%) \\
\hline A & 45.34 & 51.39 & 49.64 & 82.35 & 71.12 & 81 & 6 & 63.47 \\
B & 56.97 & 34.83 & 42.72 & 80.22 & 69.23 & 64.79 & 6 & 58.13 \\
C & 71.64 & 76.2 & 73.98 & 71.66 & 70.77 & 72.6 & 6 & 72.81 \\
D & 5.47 & 5.04 & 0 & 0 & 0 & 5.3 & 6 & 6.64 \\
E & 4.55 & 5.54 & 4.34 & 3.85 & 5.26 & 6.15 & 6 & 4.95 \\
F & 3.79 & 4.07 & 3.22 & 4.52 & 5.09 & 7.23 & 6 & 4.65 \\
\hline
\end{tabular}

AML, acute myeloid leukemia.

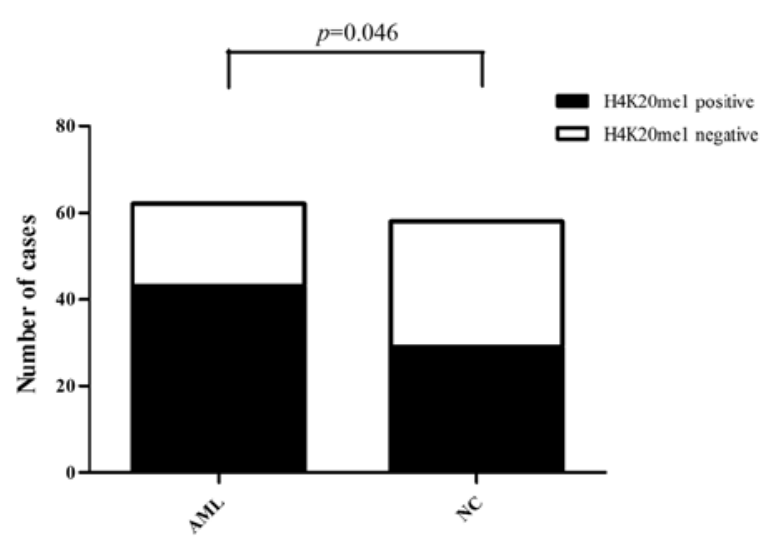

Figure 6. H4K20me1 expression is significantly higher in AML patients than in normal controls (Pearson Chi-square value $=4.677, \mathrm{P}<0.05$ ). AML, acute myeloid leukemia.

the expression of H4K20me1 was significantly higher in the AML patients than that in the normal controls (Pearson Chi-square value $=4.677, \mathrm{P}<0.05)$ (Fig. 6).

Correlation of Wnt5a and H4K20mel expression, and the methylation rate of the Wnt5a promoter in bone marrow mononuclear cells from AML patients. Correlation analysis of the gray-scale values of H4K20me1 and Wnt5a bands revealed that $\mathrm{Wnt} 5 \mathrm{a}$ expression was positively correlated with H4K20me1 expression in the AML samples; that is, high H4K20mel expression was associated with high Wnt5a expression (Pearson correlation coefficient $=0.843 ; \mathrm{P}<0.05$ ). In the normal controls, Wnt5a expression was unrelated to H4K20me1 expression (Pearson correlation coefficient $=-0.239$; P>0.05) (Fig. 7).

Correlation analysis of the methylation rate of the Wnt5a promoter (pyrophosphate sequencing) and Wnt5a and H4K20me1 protein expression (western blotting) revealed that Wnt5a and H4K20mel expression was highly concordant and positively correlated in the AML samples. However, Wnt5a expression and promoter methylation was not necessarily concordant; Wnt5a expression was detected in all AML samples with a negative methylation status (methylation rate $<10 \%$ ), but in AML samples with a positive methylation status (methylation rate $\geq 10 \%$ ), Wnt5a expression (and
Table III. The relationship among the methylation rate of the Wnt5a promoter and Wnt5a and H4K20me1 expression.

\begin{tabular}{lcccc}
\hline & $\begin{array}{c}\text { Disease } \\
\text { type }\end{array}$ & $\begin{array}{c}\text { Mean methylation } \\
\text { rate (\%) }\end{array}$ & Wnt5A & H4K20me1 \\
\hline P13 & M5 & 0.00 & + & + \\
P14 & M2 & 3.19 & + & + \\
P15 & M1 & 3.42 & + & + \\
P16 & M5 & 4.25 & + & + \\
P17 & M5b & 5.04 & + & + \\
P18 & M3 & 7.70 & + & + \\
P19 & M3 & 10.69 & - & - \\
P20 & M5b & 14.54 & + & + \\
P21 & M3 & 18.29 & - & - \\
P22 & M1 & 19.84 & + & + \\
P23 & M2 & 20.27 & - & - \\
P24 & M5 & 26.58 & + & + \\
P25 & M3 & 30.31 & - & - \\
P26 & M2 & 50.53 & + & + \\
P27 & M5 & 55.18 & + & + \\
P28 & M0 & 72.07 & + & + \\
\hline & & & + \\
\hline
\end{tabular}

H4K20me1 expression) was negative in samples with an inactivation of promoter methylation and was positive in samples with promoter methylation (Table III).

H4K20mel and SET8 enrichment in the Wnt5 a promoter in bone marrow cells from AML patients compared with normal controls. ChIP-qPCR was performed to assess H4K20mel and SET8 enrichment in the Wnt5a promoter and coding regions of normal controls and the AML samples that displayed methylation of the Wnt5a promoter. The results showed that the H4K20me1 and SET8 enrichment degree was significantly higher in the Wnt5a promoter and coding regions of AML samples with a relatively high Wnt5a expression (Wnt5a band on western blotting) compared with both normal controls and AML cases with a relatively low Wnt5a expression (no Wnt5a 


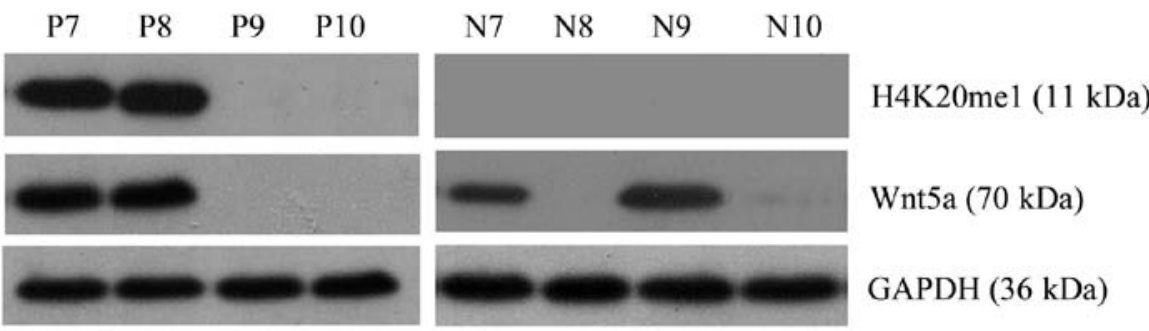

Figure 7. Correlation analysis of the gray-scale values of H4K20me1 and Wnt5a bands reveals that Wnt5a expression is positively correlated with H4K20me1 expression in the AML samples; Wnt5a expression is unrelated to H4K20me1 expression in the normal controls; N7-N10, normal controls; P7-P10, AML. AML, acute myeloid leukemia.

A

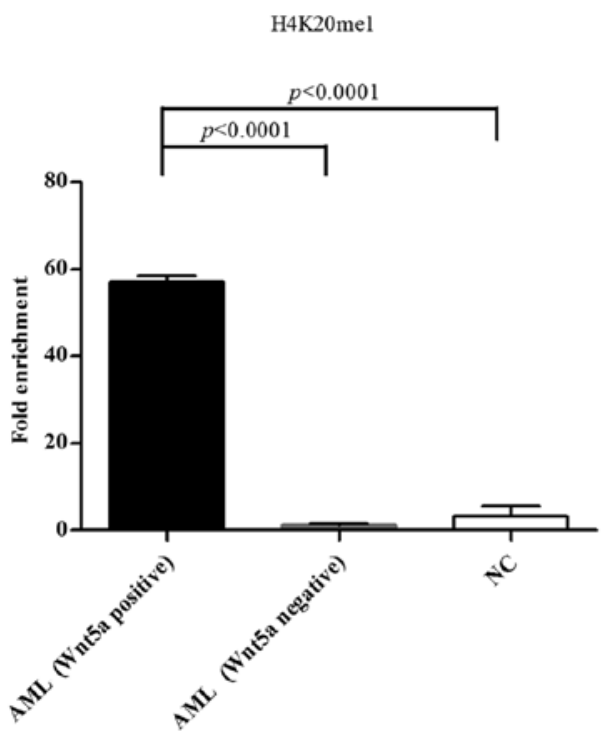

$\mathrm{C}$

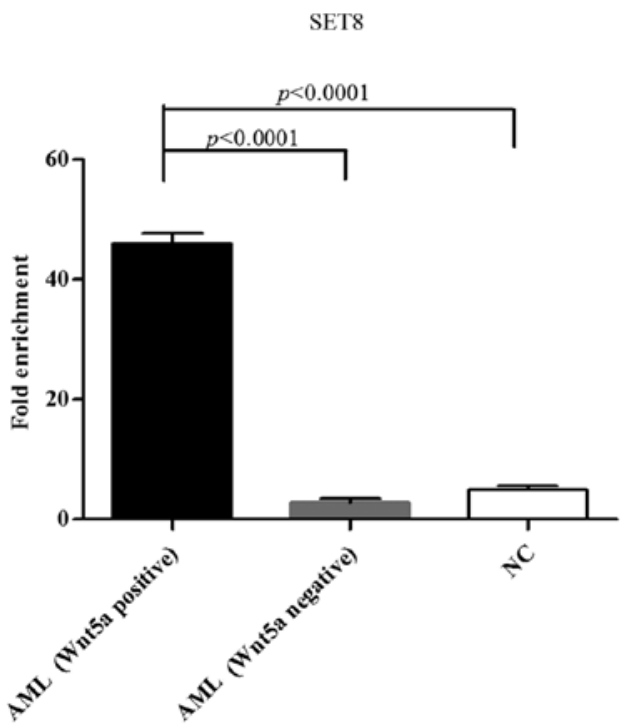

B

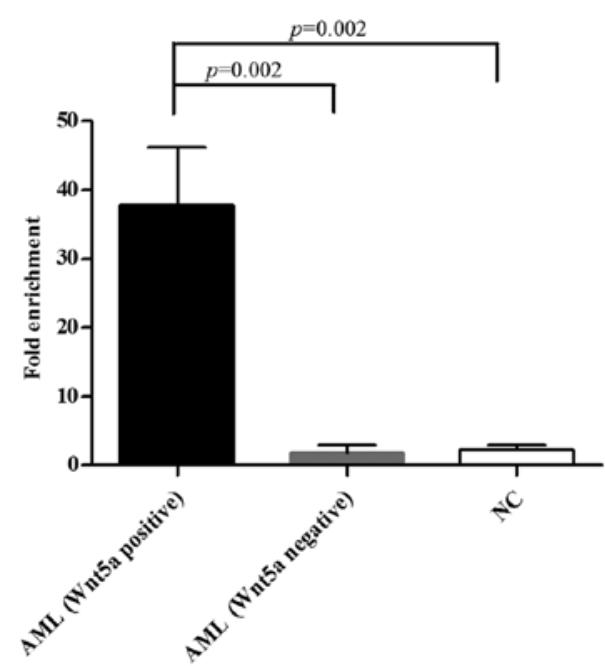

$\mathrm{D}$

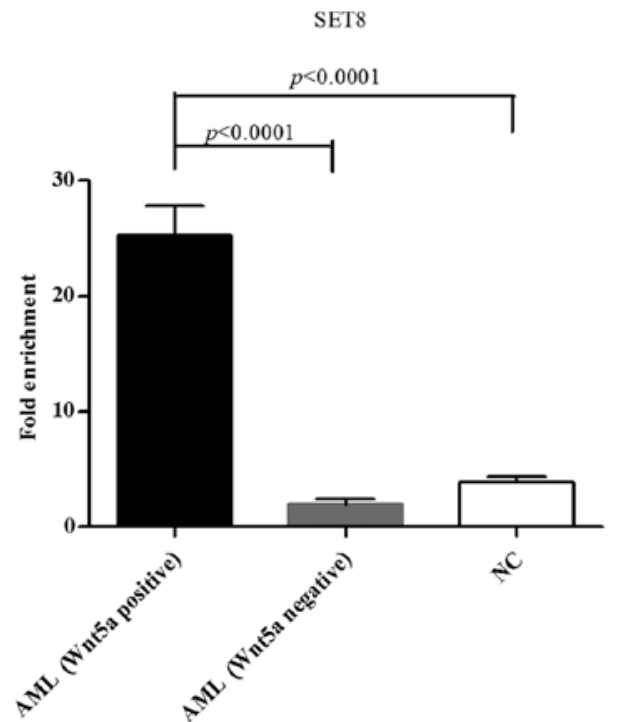

Figure 8. H4K20me1 and SET8 enrichment degree is significantly higher in the Wnt5a promoter and coding regions of the AML samples with a relatively high Wnt5a expression (Wnt5a band on western blotting) compared with both the normal controls and the AML cases with a relatively low Wnt5a expression (no Wnt5a western blot band); these differences are significant (both $\mathrm{P}<0.05$ ). (A) H4K20me1 enrichment degree in the Wnt5a promoter in the AML patients with relatively high Wnt5a expression was significantly higher than that in low Wnt5a AML and normal controls. (B) H4K20me1 enrichment degree in the Wnt5a coding region in the AML patients with relatively high Wnt5a expression was significantly higher than that in low Wnt5a AML and normal controls. (C) SET8 enrichment degree in the Wnt5a promoter in the AML patients with relatively high Wnt5a expression was significantly higher than that in low Wnt5a AML and normal controls. (D) SET8 enrichment degree in the Wnt5a coding region in the AML patients with relatively high Wnt5a expression was significantly higher than that in low Wnt5a AML and normal controls. AML, acute myeloid leukemia. 
western blot band); these differences were significant (both $\mathrm{P}<0.05$ ) (Fig. 8).

\section{Discussion}

Acute leukemia (AL) is a malignant hematopoietic tumor that ranks as the sixth most common tumor in China. The cause of AL is unknown. Studies have shown that in addition to cytogenetic changes, the expression of one or more tumor-suppressor genes related to DNA methylation, histone modifications or RNA-related silencing is often absent in leukemia. Epigenetic silencing decreases or eliminates the expression of tumor-suppressor genes in leukemia cells, which may be an alternative mechanism for genetic variation.

Methylation of $\mathrm{CpG}$ islands in the promoters of Wnt antagonists contributes to the development of hematologic malignancies, such as ALL, AML, chronic lymphocytic leukemia and myeloproliferative neoplasms (9-13). Tolwinski and Wieschaus (10) examined samples from a group of young patients with de novo AML and a moderate-risk karyotype and found that the methylation of $6 \mathrm{Wnt}$ antagonists (including sFRP1, sFRP2, sFRP4, sFRP5, DKK1 and DKK3) was associated with poor outcomes in these patients. Given the role of unusual hypermethylation of the Wnt pathway signaling molecules and the absence of their expression in the development of AL, we studied the Wnt pathway in the present study.

Wnt5a can activate or inhibit downstream pathways in both classical and non-canonical Wnt signaling. These dichotomous roles may be attributed to the protumor or antitumor effect of Wnt5a,tumor type and stage, and expression of cell surface receptors $(14,15)$. For example, Wnt5a is considered an oncogene in breast cancer and melanoma. However, in colorectal and thyroid cancer, ALL and AML, Wnt5a is considered a tumor-suppressor gene $(16,17)$. Perner et al (4) determined the methylation status of the Wnt5a promoter to the first exon in 252 AML samples using MSP and sequencing and found that the methylation rate was $43 \%$. Low Wnt5a expression was correlated with methylation and Wnt5a hypermethylation was correlated with high cyclin D1 expression. For patients with negative methylation, the relapse and mortality rates were low, the disease-free survival (DFS) rate was $60 \%$, and the 6- to 7-year overall survival (OS) was $27 \%$. For patients with hypermethylation, the DFS and OS rates were 20 and $0 \%$, respectively.

Studies in colorectal cancer showed that in addition to Wnt5a methylation, more histone modifications, including low expression of histone $\mathrm{H} 3$ and $\mathrm{H} 4, \mathrm{H} 3 \mathrm{~K} 4 \mathrm{me} 2$ acetylation and high H3K27me 3 expression in the promoter region, were observed in the colorectal cancer cell line SW620, which has low Wnt5a expression, compared with SW480 cells (17). However, the relationship between Wnt5a and histone modifications during the development of AL has not been studied. Our previous study showed that $\mathrm{H} 3 \mathrm{~K} 9 \mathrm{me} 3$ was enriched in the Wnt5a promoter in the ALL cell line Jurkat. This histone modification inhibited gene transcription and provided the rationale for us to further investigate histone modifications of the Wnt5a promoter.

The present study showed that the mRNA expression levels of Wnt antagonists (Wnt5a, HDPR1, DKK1 and DKK3) were significantly lower in the AML patients than those in the normal controls and had a differential expression in the
ALL patients (low Wnt5a expression in ALL-T, but normal Wnt5a expression in ALL-B). The expression of DKK3 was lower in the ALL patients than that in the normal controls. The expression of HDPR 1 was comparable between the ALL patients and the normal controls. The expression of DKK1 was significantly higher in the ALL patients than that in the normal controls. The differential expression in ALL may be used to diagnose different types of leukemia; in particular, the high DKK1 expression in the ALL patients and the low DKK1 expression in the AML patients may be used as alternative diagnostic biomarkers.

Pyrophosphate sequencing revealed that the methylation rate of the Wnt5a promoter was significantly higher in the AML patients compared with that in the normal controls. Western blotting showed that overall, Wnt5a expression was significantly lower in the AML patients than in the normal controls, which was consistent with the real-time fluorescence qPCR results and largely consistent with pyrophosphate sequencing results. Most samples with a high methylation rate exhibited low protein expression. These results suggest that promoter methylation is the main cause of the low expression of Wnt antagonists and that inactivation of the methylation of tumor suppressor genes may be closely related to the development of AML, which is consistent with the results of other international studies. However, some samples with hypermethylation of the Wnt5a promoter did not exhibit low protein expression, refuting the aforementioned theory.

Furthermore, western blotting confirmed that overall, the expression of H4K20me1 was significantly higher in the AML patients than that in the normal controls and that H4K20me1 and Wnt5a expression were positively correlated in AML patients (no such correlation was observed in the normal controls). Analysis of the methylation rate of the Wnt5a promoter and H4K20me1 and Wnt5a protein expression in the AML patients revealed that all patients with a negative methylation status $(<10 \%)$ exhibited Wnt5a and H4K20me1 expression, whereas in patients with positive methylation $(\geq 10 \%)$, some were negative for both Wnt5a and H4K20me1 expression and some were positive for both Wnt5a and H4K20me1 expression. ChIP-qPCR was performed to determine H4K20mel and SET8 enrichment in the Wnt5a promoter and coding regions in the AML samples that exhibited methylation of the Wnt5a promoter. The results revealed that the H4K20me1 and SET8 enrichment factor was significantly higher in the AML samples with a relatively high Wnt5a expression than in both the normal controls and AML samples with a relatively low Wnt5a expression. These data suggest that Wnt5a expression is affected by more than one methylation mechanism in AML. In the absence of H4K20me1 and SET8, promoter methylation is associated with a low expression level; with H4K20me1 and SET8 enrichment in the promoter and coding regions, transcription is activated in patients with methylation, as SET8 promotes H4K20 methylation and H4K20mel promotes transcription, offsetting the inhibitory effect of methylation on transcription. Furthermore, a higher level of H4K20me1 enrichment was associated with higher Wnt5a expression; this correlation is consistent with the theory that H4K20me1 promotes transcription initiation and elongation and provides additional information to the conventional theory that methylation inactivates gene expression. Therefore, although 
promoter methylation inhibits overall gene expression, H4K20me1 may play a larger role in gene expression than methylation. Collectively, we propose a new theory: Wnt5a (a Wnt pathway antagonist gene) expression is correlated with H4K20me1 expression, but not necessarily with methylation, in AML patients. Further research is needed to verify this theory. Moreover, in-depth research is needed to investigate why some patients with Wnt5a methylation exhibit Wnt5a expression while others do not, whether H4K20me1 binding is selective, and whether these results can be applied to the methylation of other promoters. We did not observe these trends in normal controls, likely since the overall H4K20mel expression was low in these samples and since H4K20me1 is not a key regulatory histone of Wnt5a expression in normal tissues. Due to the significant differential expression of H4K20me1 between normal controls and AML patients, H4K20me1 may be used as a diagnostic biomarker.

These results confirmed widespread changes in the methylation of Wnt antagonists in AML patients and leukemia cell lines, which may be an important mechanism for the development of leukemia. Wnt5a, DKK1 and DKK3 may be potential treatment targets of malignant hematological tumors, including $\mathrm{AL}$, and may also be useful as diagnostic biomarkers for the diagnosis of and efficacy screening in these diseases. Moreover, H4K20me1 and Wnt5a expression levels were positively correlated, and this relationship was not affected by changes in promoter methylation, refuting the conventional theory that promoter methylation inhibits gene expression. Further research is needed to investigate the relationship between H4K20mel and gene expression.

\section{Acknowledgements}

The present study was supported by the Fujian Provincial Health Bureau Youth Research Project (2010-1-12), the National Natural Science Foundation of China (nos. 81370629 and 81300428), the Fujian Key Laboratory of Hematology Fund (2009J1004), the National and Fujian Clinical Key Specialist Construction Project, and the Youth Innovation Project of Natural Science Foundation of Fujian Province (2017J05132).

\section{References}

1. DeLange RJ, Fambrough DM, Smith EL and Bonner J: Calf and pea histone IV. II. The complete amino acid sequence of calf thymus histone IV; presence of epsilon-N-acetyllysine. J Biol Chem 244: 319-334, 1969.
2. Zhang Y and Reinberg D: Transcription regulation by histone methylation: Interplay between different covalent modifications of the core histone tails. Genes Dev 15: 2343-2360, 2001.

3. Oda H, Okamoto I, Murphy N, Chu J, Price SM, Shen MM, Torres-Padilla ME, Heard E and Reinberg D: Monomethylation of histone H4-lysine 20 is involved in chromosome structure and stability and is essential for mouse development. Mol Cell Biol 29: 2278-2295, 2009.

4. Perner J, Lasserre J, Kinkley S, Vingron M and Chung HR: Inference of interactions between chromatin modifiers and histone modifications: From ChIP-Seq data to chromatin-signaling. Nucleic Acids Res 42: 13689-13695, 2014.

5. Hori T, Shang WH, Toyoda A, Misu S, Monma N, Ikeo K, Molina O, Vargiu G, Fujiyama A, Kimura H, et al: Histone H4 Lys 20 monomethylation of the CENP-A nucleosome is essential for kinetochore assembly. Dev Cell 29: 740-749, 2014.

6. Yao L, Li Y, Du F, Han X, Li X, Niu Y, Ren S and Sun Y: Histone H4 Lys 20 methyltransferase SET8 promotes androgen receptor-mediated transcription activation in prostate cancer. Biochem Biophys Res Commun 450: 692-696, 2014.

7. Nishioka K, Rice JC, Sarma K, Erdjument-Bromage H, Werner J, Wang Y, Chuikov S, Valenzuela P, Tempst P, Steward R, et al: PR-Set7 is a nucleosome-specific methyltransferase that modifies lysine 20 of histone $\mathrm{H} 4$ and is associated with silent chromatin. Mol Cell 9: 1201-1213, 2002.

8. Veloso A, Kirkconnell KS, Magnuson B, Biewen B, Paulsen MT, Wilson TE and Ljungman M: Rate of elongation by RNA polymerase II is associated with specific gene features and epigenetic modifications. Genome Res 24: 896-905, 2014.

9. Figueroa ME, Skrabanek L, Li Y, Jiemjit A, Fandy TE, Paietta E, Fernandez H, Tallman MS, Greally JM, Carraway H, et al: MDS and secondary AML display unique patterns and abundance of aberrant DNA methylation. Blood 114: 3448-3458, 2009.

10. Tolwinski NS and Wieschaus E: A nuclear function for armadillo/ $\beta$-catenin. PLoS Biol 2: E95, 2004.

11. Claus R, Almstedt M and Lübbert M: Epigenetic treatment of hematopoietic malignancies: In vivo targets of demethylating agents. Semi Oncol 32: 511-520, 2005.

12. Picard F, Cadoret JC, Audit B, Arneodo A, Alberti A, Battail C, Duret L and Prioleau MN: The spatiotemporal program of DNA replication is associated with specific combinations of chromatin marks in human cells. PLoS Genet 10: e1004282, 2014.

13. Bannister AJ, Schneider R and Kouzarides T: Histone methylation: Dynamic or static? Cell 109: 801-806, 2002.

14. Schübeler D, MacAlpine DM, Scalzo D, Wirbelauer C, Kooperberg C, van Leeuwen F, Gottschling DE, O'Neill LP, Turner BM, Delrow J, et al: The histone modification pattern of active genes revealed through genome-wide chromatin analysis of a higher eukaryote. Genes Dev 18: 1263-1271, 2004.

15. Liang G, Lin JCY, Wei V, Yoo C, Cheng JC, Nguyen CT, Weisenberger DJ, Egger G, Takai D, Gonzales FA, et al: Distinct localization of histone $\mathrm{H} 3$ acetylation and H3-K4 methylation to the transcription start sites in the human genome. Proc Natl Acad Sci USA 101: 7357-7362, 2004.

16. Ysebaert L, Chicanne G, Demur C, De Toni F, Prade-Houdellier N, Ruidavets JB, Mansat-De Mas V, Rigal-Huguet F, Laurent G, Payrastre B, et al: Expression of $\beta$-catenin by acute myeloid leukemia cells predicts enhanced clonogenic capacities and poor prognosis. Leukemia 20: 1211-1216, 2006.

17. Shi Y, Lan F, Matson C, Mulligan P, Whetstine JR, Cole PA, Casero RA and Shi Y: Histone demethylation mediated by the nuclear amine oxidase homolog LSD1. Cell 119: 941-953, 2004. 